\title{
Preparation of Novel Nonlinear Optical Polyester with Enhanced Thermal Stability of Dipole Alignment
}

\author{
Ju-Yeon Lee," Jin-Hyang Kim, Dong-Seon Won, and Han-Na Jang \\ Institute of Functional Materials, Department of Chemistry, Inje University. Gimhae 621-749, Korea \\ "E-mail: chemljyainieackr \\ Received April 28,2007
}

Key Words : Nonlinear optics, Polyester. Thermal stability, Second harmonic generation, Relaxation of dipole alignment

Nonlinear optical (NLO) polymers have been extensively studied over the past decade because of their potential applications in the field of electro-optic devices. ${ }^{1-3} \Lambda$ potential NLO polymer have to be mechanically very strong and thermally stable with high glass-transition temperatures $\left(T_{g}\right)$. In the developments of NLO polymers for electro-optic device applications, stabilization of electrically induced dipole alignment is important considerations. Two approaches to minimize the randomization of dipole alignment have been proposed. One is to use crosslinking method ${ }^{4-6}$ and the other is to utilize high $T_{p}$ polymers such as polyimides. ${ }^{7}$ Polyesters with the NLO-chromophores in the main chatin or in side chain ${ }^{8}$-11 were prepared and investigated. Main-chain NI.O polymers have good thermal stability of dipole alignments, but they often do not dissolve in organic solvents, and the intractability of them make them impossible to fabricate stable noncentrosymmetric films. Side-chain NI O polymer systems have the advantages such as good solubility, homogeneity and high level of N1.O chromophore relative to the main-chain systems, but they often suffer from poor stability of dipole alignments at high temperatures. Recently we have prepared polyesters containing dioxynitrostilbenyl group as a NLO chromophore, , $2.13^{3}$ The resulting polymers exhibited thermal stability of second harmonic generation (SHG) up to $T_{\mathrm{s} .}$. This high thermal stability of optical nonlinearity stemmed from the stabili-

(a)

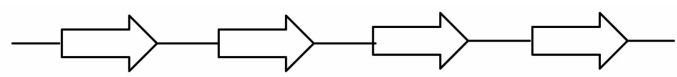

(b)

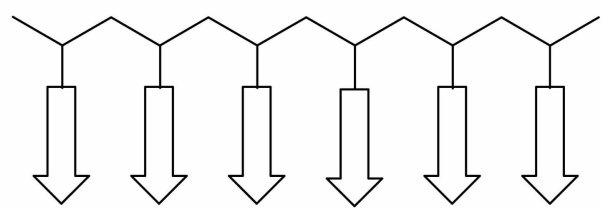

(c)

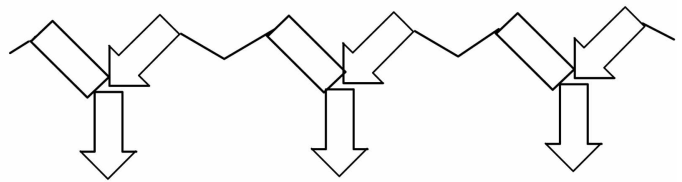

Figure 1. (a) Main-chain NI.O polymers, (b) side-chain NLOO polymers and (c) Y-lype NI,O polymers. zation of dipole alignment of the N1.O chromophore, which was a part of the polymer backbone. In this work we prepared novel polyester containing 2,3-dioxybenzylidenemalononitrile groups as NI.O-chromophores. We selected 2,3-dioxybenzylidenemalononitrile groups as NI O-chromophores because they have a large dipole moment and are rather easy to synthesize. Furthermore, 2,3-dioxybenzylidenemalononitrile group constitutes novel Y-type NI.O polyester ( $\Gamma i g .1 \mathrm{c}$ ), and this Y-type NI.O polyester has not been reported in the literature. These mid-type NI.O polymers are expected to have the advantages of both main-chain and side-chain N1.O polymers: stabilization of dipole alignment and good solubility. After confirming the structure of the resulting polymers we investigated the properties such as solubility, $T_{\mu}$, thermal stability, second harmonic generation (SHG) activity and relaxation of dipole alignment. We now report the results of the initial phase of the work.

\section{Results and Discussion}

2,3-Di-(2'-vinyloxyethoxy)benzaldehyde (1) was prepared by the reaction of 2-chlorocthyl vinyl ether with $2.3-$ dihydroxybenaldehyde. 2,3-Di-(2'-vinyloxyethoxy)benzylidenemalononitrile (2) was prepared by the condensation reaction of 1 with malononitrile. Compound 2 was hydrolyzed to yield acetaldehyde and diol 3 . Diol 3 was condensed with terephthaloyl chloride in a dry DMF solvent to yield polyester 4 containing the NLO-chromophore 2,3-dioxybenzylidenemalononitrile group The synthetic route for polyeater 4 is presented in Scheme 1. The polymerization yield was $88-92 \%$. The chemical structures of the resulting polymers were confirmed by 'H NMR, IR spectra, and elemental analysis. Elemental analysis results fit the polymer structures. ${ }^{1} \mathrm{H}$ NMR spectra of the polymers showed a signal broadening due to polymerization, but the chemical shifts are consistent with the proposed polymer structures. The IR spectra of the same polymer samples show a strong carbonyl peak near $1722 \mathrm{~cm}^{1}$ indicating the presence of ester bond. The same polymer sample also shows a strong absorption peak near $2229 \mathrm{~cm}^{-1}$ and $1572 \mathrm{~cm}^{-1}$ due to the nitrile and olefin groups, respectively. These results are consistent with the proposed structures, indicating that the NLO-chromophore remained intact during the polymeri- 


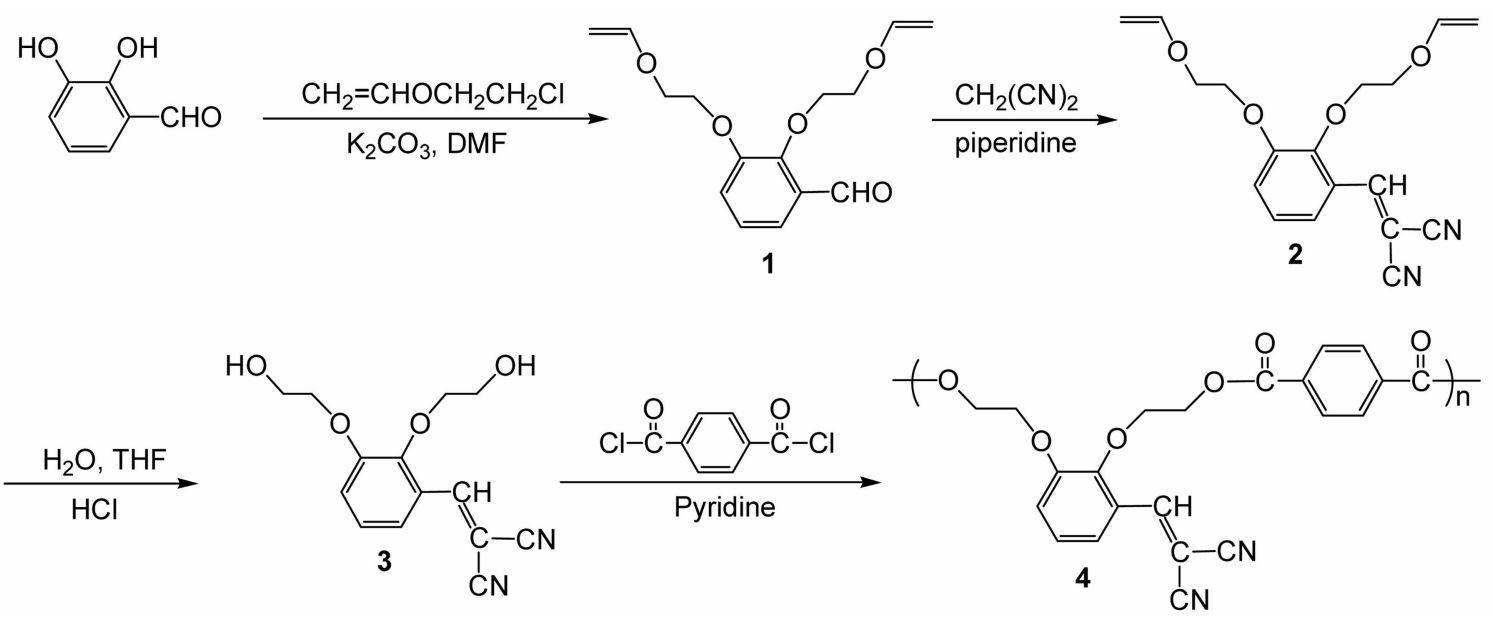

Scheme 1. Synthetic scheme and structure of polymer 4.

zation. The molecular weights were determined by GPC using polystyrene as the standard and $\mathrm{THF}$ as an eluent. The number average molecular weight $\left(M_{n}\right)$ of the polymers was determined to be $16800\left(M_{\mathrm{v}} / M_{\mathrm{n}}=1.86\right)$ for polymer 4 . Polydispersities were in the range of 1.84-1.95. The structural feature of these polymers is that they have pendant NLO chromophores, which are parts of the polymer main chains. Thus the resulting polymer (4) is a mid type of side chain- and main chain NLO polymer, and is expected to have both of their merits. The polymer $\mathbf{4}$ was soluble in common solvents such as acetone, DMF, and DMSO, but was not soluble in methanol and diethyl ether. Polymer 4 isolated from methanol were yellow colored amorphous materials. The inherent viscosities were in the range of of $0.28-0.30 \mathrm{dL} / \mathrm{g}$. Polymer 4 showed strong absorption near $340 \mathrm{~nm}$ by the NLO-chromophore dioxybenzylidenemalononitrile group. We now have well defined polyester (4) and investigate the properties.

The thermal behavior of the polymers was investigated by thermogravimetric analysis ([GA) and differential scanning calorimeter (DSC) to determine the thermal degradation

Table 1, 'l hermal Properties of Polymer 4

\begin{tabular}{|c|c|c|c|c|c|}
\hline \multirow{2}{*}{ Polymer } & \multirow{2}{*}{$\begin{array}{l}T_{\mathrm{H}} \cdot \\
{ }^{\prime \prime} \mathrm{C}\end{array}$} & \multicolumn{3}{|c|}{ Degradation temp. "C." } & \multirow{2}{*}{$\begin{array}{l}\text { Residue at } \\
800^{\circ} \mathrm{C} . \%\end{array}$} \\
\hline & & $5 \%$ moss & $20 \%$-loss & $40 \%$-loss & \\
\hline 4 & 90 & 306 & 392 & 468 & 42.2 \\
\hline
\end{tabular}

"Detemined from DSC curves measured on a TA 2920 differential scaminge calorimeter with a heating rate of $10^{\circ} \mathrm{C}$ imin under nilroger atmosphere. "Determined from TCA curves measured on a DuPont 951 thermogravimetric analyzer with a heating rate of $10^{\circ} \mathrm{C} / \mathrm{min}$ under nitrogen atmosphere. pattern and glass transition temperature $\left(I_{0}\right)$. The results are summarized in Table 1. Polymer $\mathbf{4}$ showed a thermal stability up to $290{ }^{\circ} \mathrm{C}$ according to their TGA thermograms. The initial weight loss in the polymers begins at $278^{\circ} \mathrm{C}$. The $T_{s}$ value of the polymer 4 measured by DSC were around $90^{\circ} \mathrm{C}$.

The NLO properties of polymers were studied by the SHG method. To induce noncentrosymmetric polar order, the spin-coated polymer films were corona-poled. The UV-Vis absorption spectra of the polymer sample $\mathbf{4}$ before and after the poling are recorded. After the electric poling, the dipole moments of the NLO-chromophores were aligned and UV$V$ is spectrum of polymer $\mathbf{4}$ exhibited a slight blue shift and a decrease in absorption due to birefringence. From the absorbance change, the order parameter of the poled film could be estimated, which is related to the poling efficiency. The estimated order parameter value $\Phi$ was found to have a value of 0.23 for polymer $4\left(\Phi=1-A_{1} / A_{1}\right)$, where $A_{0}$ and $A_{1}$ are the absorbances of the polymer film before and after poling). The refractive index of the sample was measured by the optical transmission technique. ${ }^{14} \mathrm{SHG}$ measurements were performed at a fundamental wavelength of $1064 \mathrm{~nm}$ using a mode locked Nd-YAG laser. NLO properties of polymer $\mathbf{4}$ are summarized in Table 2 . In order to determine the microscopic second-order susceptibility of the polymer. the angular SHG dependence was recorded. Figure 2 shows the angular dependence of SHG signal in a poled polymer 4. The SHG values were compared with those obtained from a Y-cut quartz plate. Jo calculate the $d_{31}$ and $d_{s}$ values, both $s$ polarized and $p$-polarized IR laser were directed to the samples and recorded. SHG coefficients $\left(d_{3 i}\right)$ were derived from the analysis of measured Maker-fringes with Pascal

Table 2. Nonlinear Optical Properties of Polymer 4

\begin{tabular}{|c|c|c|c|c|c|c|}
\hline J'olymer & $\hat{\lambda}_{\text {matax }}{ }^{a}(\mathrm{~nm})$ & $d_{33}^{b}(\mathrm{esu})$ & $\Phi{ }^{\prime \prime}$ & $d_{31}{ }^{h}(\mathrm{esu})$ & film thickness ${ }^{t}(L \mathrm{~mm})$ & $n$ \\
\hline 4 & 340 & $(3.52 \pm 0.10) \times 10^{-9}$ & 0.23 & $(1,43 \pm 0.04) \times 10^{-9}$ & 0.54 & $\begin{array}{l}n_{1}-1.60 \\
n_{2}=1.68\end{array}$ \\
\hline
\end{tabular}

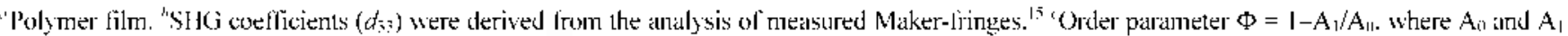
are the absorbances of the polymer film before and after corona poling. respectively. "Film thichness was determined by the optical transmission technique..". 


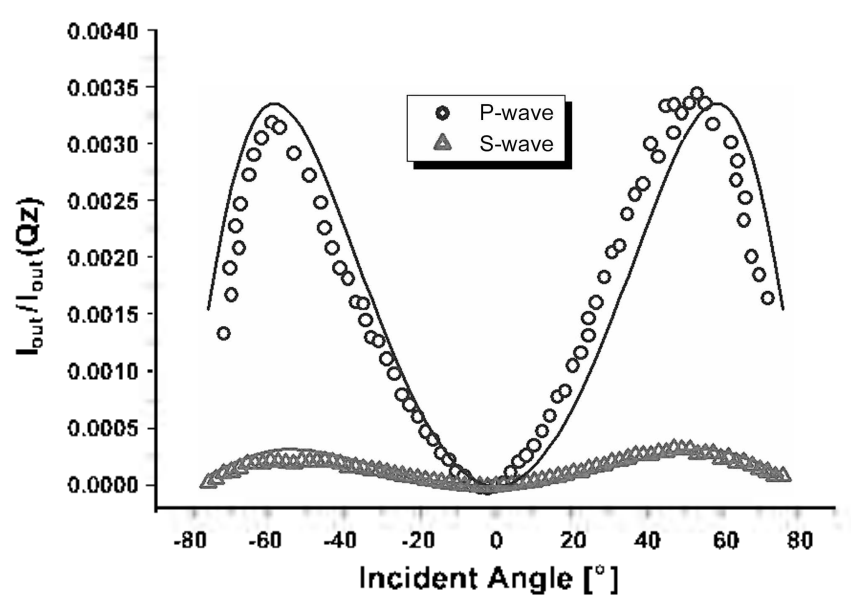

Figure 2. Angular dependence of $\mathrm{SHG}$ signal in a poled film of polymer 4.

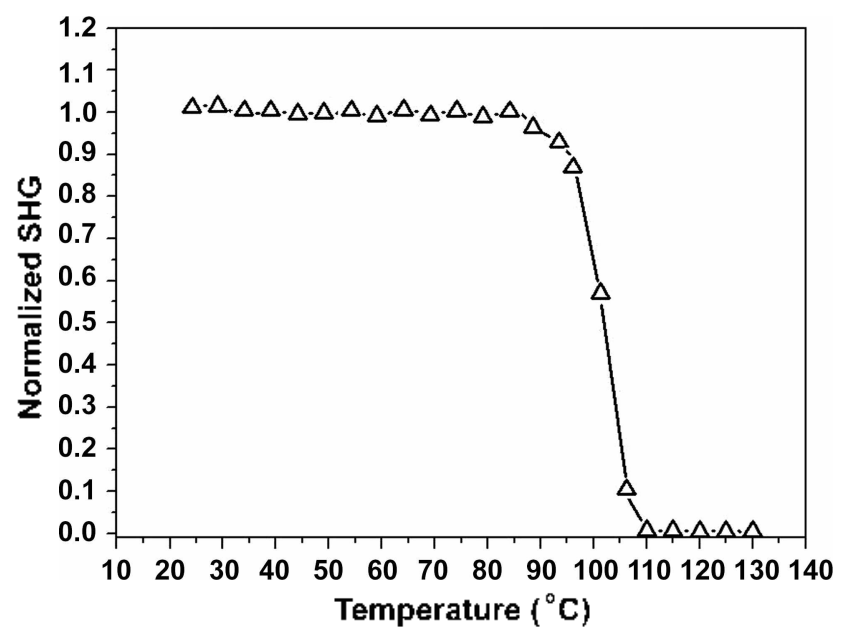

Figure 3. Normalized SI IG signal ol polymer 4 as a function of temperalure at at heating rale of $3^{\circ} \mathrm{C} / \mathrm{min}$.

fitting program according to the literature procedure. ${ }^{15}$ The values of $d_{3}$ and $d_{31}$ for polymer 4 were $(3.52 \pm 0.10) \times 10^{-4}$ and $(1.43=0.04) \times 10^{-9}$ esu, respectively. Since the second harmonic wavelength was at $532 \mathrm{~nm}$, which is not in the absorptive region of the resulting polyesters, there was not resonant contribution to this $d_{3: 3}$ value. To evaluate the hightemperature stability of the polymers, we studied the temporal stability of the SHG signal. In Figure 3, we present the dynamic thermal stability study of the NLO activity of the film 4. To investigate the real time NLO decay of the SHG signal of the poled polymer films as a function of temperature, in situ SHG measurements were performed at a heating rate of $3{ }^{\circ} \mathrm{C} / \mathrm{min}$ from $30^{\circ} \mathrm{C}$ to $150^{\circ} \mathrm{C}$. The polymer film exhibited a thermal stability up to $T_{\mu}$ and no significant SHG decay was observed below $95^{\circ} \mathrm{C}$, as shown in Figure 3. In general, side chain NLO polymers lose thermal stability of dipole alignment around $T_{j \cdot}$. Stabilization of dipole alignment is a characteristic of main chain NLO polymers. The high thermal stability of second harmonic generation of polymer 4 is due to the stabilization of dipole alignment of NLO chromophore, which stems from the partial main chain character of the polymer structure. Thus, we obtained a new type of NLO polyester having both of the merits of main chain- and side-chain NLO polymers: stabilization of dipole alignınent and good solubility.

\section{Experimental Section}

Materials. The reagent grade chemicals were purchased from Aldrich and purified by either distillation or recrystallization before use. 2,3-Dihydroxybenzaldehyde, 2-chloroethyl vinyl ether, and tetrahydrofuran (THF) were used as received. Terephthaloyl chloride (TPC) was purified by sublimation under vacuum. Malononitrile was recrystallized from water and distilled under reduced pressure. Piperidine was treated with potassium hydroxide and then distilled over barium oxide to remove trace amounts of water. $N N$ Dimethylformamide (DMF) was purified by drying with anhydrous calcium sulfate, followed by distillation under reduced pressure.

Film Preparation and SHG Measurements. The refractive index of the sample was measured by the optical transmission technique. ${ }^{1.4}$ The transmittance of thin film includes on the information of the thickness, refractive index and extinction coefficient of that. Thus, we determined these parameters by analyzing the transmittance. Second harmonic generation (SHG) measurement was carried out one day after poling. The Maker Fringe pattern was obtained from measuring the SHG signal at $0.5^{\circ}$ intervals using a rotation stage. SHG coefficients $\left(d_{3}\right)$ were derived from the analysis of measured Maker-fringes. ${ }^{15}$

Preparation of 2,3-(2'-Vinyloxyethoxy)benzaldehyde (1). 2.3-Dihydroxybenzaldehyde (13.8 g. $0.10 \mathrm{~mol})$, anhydrous potassium carbonate ( $36 \mathrm{~g}, 0.36 \mathrm{~mol})$, and 2-chloroethyl vinyl ether (26.6 g. $0.25 \mathrm{~mol}$ ) were dissolved in $200 \mathrm{~mL}$ of dry DMF under nitrogen. The mixture was refluxed in an oil bath kept at $100{ }^{\circ} \mathrm{C}$ for $40 \mathrm{~h}$ under nitrogen. The resulting solution was cooled to room temperature, diluted with 300 $\mathrm{mL}$ of water, and extracted with $300 \mathrm{~mL}$ of diethyl ether three times. The organic layer was washed with saturated aqueous sodium chloride solution, and dried with anhydrous magnesiuın sulfate. Rotary evaporation of diethyl ether gave crude product, which was recrystallized from 1-butanol yielded $24.5 \mathrm{~g}$ ( $88 \%$ yield) of pure product $\mathrm{I} . \mathrm{Mp}=48-50$ ${ }^{\circ} \mathrm{C}$. ${ }^{1} \mathrm{H}$ NMR $\left(\mathrm{CDCl}_{3}\right) \delta 3.95-4.47\left(\mathrm{~m}, 12 \mathrm{H}, 2 \mathrm{CH}_{2}=.2-\mathrm{O}-\right.$ $\left.\mathrm{CH}_{2}-\mathrm{CH}_{2}-\mathrm{O}-\right)$, 6.44-6.57 (m, 2H, $\left.2=\mathrm{CH}-\mathrm{O}-\right), 7.09-7.19(\mathrm{~m}$, $2 \mathrm{H}$, aromatic), 7.42-7.48 (m, $1 \mathrm{H}$, aromatic), 10.48 (s, $1 \mathrm{H}$. $-\mathrm{CHO})$. IR (KBr) 3098, $3076(\mathrm{w},=\mathrm{C}-\mathrm{H}), 2953,2883$ (s. CH), $1682(\mathrm{vs}, \mathrm{C}=\mathrm{O}), 1618(\mathrm{vs}, \mathrm{C}=\mathrm{C}) \mathrm{cm}^{-1}$.

Preparation of 2,3-Di-(2'-vinyloxyethoxy)benzylidenemalononitrile (2). P'iperidine $(0.13 \mathrm{~g}, 1.5 \mathrm{mmol})$ was added to a solution of 2,3-di-(2'-vinyloxyethoxy)benzaldehyde 1 $(8.35 \mathrm{~g} .30 \mathrm{mmol})$ and malononitrile $(2.18 \mathrm{~g} .33 \mathrm{mmol})$ in $170 \mathrm{~mL}$ of I-butanol with stirring at $0{ }^{\circ} \mathrm{C}$ under nitrogen. After stirring for $4 \mathrm{~h}$ at $0^{\circ} \mathrm{C}$, the reaction mixture was cooled to $-10^{\circ} \mathrm{C}$ for crystallization. The product was filtered and washed successively with cold I-butanol $(80 \mathrm{~mL})$, water $(30$ $\mathrm{mL})$, and cold 1-butanol $(20 \mathrm{~mL})$. The obtained pale yellow 
product was recrystallized from 1-butanol to give $8.11 \mathrm{~g}$ (83\% yield) of $2 . \mathrm{Mp}=60.62^{\circ} \mathrm{C} .{ }^{1} \mathrm{H}$ NMR $\left(\mathrm{CDCl}_{3}\right) \delta 3.90$ $4.42\left(\mathrm{~m} . \mathrm{L} 2 \mathrm{H}, 2 \mathrm{CH}_{2}=, 2-\mathrm{O}-\mathrm{CH}_{2}-\mathrm{CH}_{2}-\mathrm{O}-\right)$, 6.45-6.57 (m. $2 \mathrm{H}, 2=\mathrm{CH}-\mathrm{O}-$ ). $7.13-7.20$ (d. $2 \mathrm{H}$. aromatic), 7.80-7.87 (t. lH, aromatic). 8.47 (s, IH. Ph-CH=). IR (KBr) 3116.3059 $(\mathrm{w},=\mathrm{C}-\mathrm{H}), 2941,2885(\mathrm{~m}, \mathrm{C}-\mathrm{H}), 2233$ (s. CN), 1618. 1572 (vs, $\mathrm{C}=\mathrm{C}$ ) $\mathrm{cm}^{-1}$. Anal. Calcd for $\mathrm{C}_{18} \mathrm{H}_{18} \mathrm{~N}_{2} \mathrm{O}_{4}: \mathrm{C} .66 .25 ; \mathrm{H}$. 5.56: N. 8.58. Found C. $66.16 ;$ H. $5.4 ;$ N. 8.65 .

Preparation of 2,3-Di-(2'-hydroxyethoxy)benzylidenemalononitrile (3). Aqueous hydrochloric acid $(1.5 \mathrm{M}, 30$ $\mathrm{mL}$ ) was slowly added to a solution of 2,3 -di-(2'-vinylosyethoxy)benzylidenemalononitrile (2) $(8.48 \mathrm{~g} .0 .026 \mathrm{~mol})$ in $60 \mathrm{~mL}$ of dry THF with stirring under nitrogen at $0{ }^{\circ} \mathrm{C}$. The mixture was stirred at $80^{\circ} \mathrm{C}$ for $8 \mathrm{~h}$ under nitrogen. The resulting solution was extracted with diethyl ether $(80 \mathrm{~mL})$ three times. The organic layer was washed successively with saturated sodium chloride, sodium hydrogen carbonate. and water, followed by drying with anlydrous magnesium sulfate. Rotary evaporation of diethyl ether gave crude product. The obtained pale yellow product was recrystallized from ethyl acetate to give $5.85 \mathrm{~g}$ ( $82 \%$ y ield) of $3 . \mathrm{Mp}=116$ $118{ }^{\circ} \mathrm{C} .{ }^{\mathrm{i}} \mathrm{H}$ NMR (acetone- $\left.d_{6}\right) \quad \delta 3.75-3.96\left(\mathrm{~m}, 4 \mathrm{H} .2-\mathrm{CH}_{5}-\right.$ $\mathrm{OH}) .4 .12-4.25\left(\mathrm{~m}, 4 \mathrm{H}, 2-\mathrm{O}-\mathrm{CH}_{2}-\right), 4.26-4.34$ (t. $2 \mathrm{H},-\mathrm{OH}$ ). $7.21-7.29(\mathrm{t}, 1 \mathrm{H}$, aromatic), 7.37-7.44 (d. $1 \mathrm{H}$. aromatic). $7.72-7.78$ (d. $1 \mathrm{H}$, aromatic), 8.75 (s. $1 \mathrm{H},-\mathrm{Ph}-\mathrm{CH}=$ ). IR $(\mathrm{KBr}) 3360,3256$ (s. O-H). 3044 (w, =C-H). $294 \mathrm{l}$ (m. C.H), 2222 (m. CN). 1572 (vs, C=C) $\mathrm{cm}^{-1}$. Anal. Calcd for $\mathrm{C}_{14} \mathrm{H}_{1} \mathrm{~N}_{2} \mathrm{O}_{4}$ : C. 61.31; H, 5.14: N. 10.21. Found: C. 61.23: H. $5.22: \mathrm{N}, 10.13$.

Synthesis of Polyester 4. A representative polycondensation procedure was as follows: Terephthaloyl chloride $(2.03 \mathrm{~g}, 0.01 \mathrm{~mol})$ and diol $3(2.74 \mathrm{~g} .0 .01 \mathrm{~mol})$ were disolved in $30 \mathrm{~mL}$ of anlydrous pyridine under nitrogen. The resulting solution was refluxed in an oil bath kept at 80 ${ }^{\circ} \mathrm{C}$ under a nitrogen atmosphere. After heating $20 \mathrm{~h}$ with stirring the resulting polymerization solution was poured into $400 \mathrm{~mL}$ of methanol. The precipitated polymer was collected and reprecipitated from DMSO into methanol. The polymer was further purified by extraction in a Soxhlet extractor with dietlyyl ether and dried under vacuum. yielding $3.68 \mathrm{~g}\left(91 \%\right.$ yield) of polymer $4: \eta_{\text {wh }}=0.29 \mathrm{dL} / \mathrm{g}$ (c, $0.5 \mathrm{~g} / \mathrm{dL}$ in DMSO at $25^{\circ} \mathrm{C}$ ). ${ }^{1} \mathrm{H}$ NMR (DMSO- $d_{6}$ ) $\delta$ $4.38-4.52$ (s, $4 \mathrm{H} .2-\mathrm{CH}_{2}=\mathrm{O}-$ ), 4.57-4.72 (s. $4 \mathrm{H}, 2 \mathrm{Ph}-\mathrm{O}-\mathrm{CH}_{2}-$ ), $7.20-7.35$ (s. $1 \mathrm{H}$. aromatic). 7.37-7.53 (s, $1 \mathrm{H}$, aromatic), 7.54-7.66 (s. $1 \mathrm{H}$, aromatic), 7.81-8.03 (d, $4 \mathrm{H}$. aromatic), 8.42-8.52 (s, lH, aromatic). IR ( $\mathrm{KBr}) 3049(\mathrm{w},=\mathrm{C}-\mathrm{H}), 2958$ (m. C-H), 2229 (s. CN). 1722 (vs. C=O). 1572 (s, C=C) $\mathrm{cm}^{-1}$. Anal. Calcd for $\left(\mathrm{C}_{22} \mathrm{H}_{16} \mathrm{~N}_{2} \mathrm{O}_{6}\right)_{n}: \mathrm{C}, 65.34: \mathrm{H} .3 .99 ; \mathrm{N}$. 6.93. Found: C. $65.43 ;$ H. 3.92 : N. 6.98 .

Acknowledgement. This work was supported by the 2006 Inje University research grant and partially by the BK21 project of the Ministry of Education.

\section{References}

1. Nolecular Nonlinear Optics Materials Plnsics and Derices; Zyss. T.. Ed.: Academic Press: Orlando. 1994.

2. Cho. M. I.: Kim. J. Y.: Kim. J. H.: Lee. S. H.: Dalton. L. R.: Choi. D. H. Bull. Korean Chent Soc. 2005. 26.77.

3. Lee. C. Park, S.-K; Yang M: Lee, N.-S.; Kim. N. J. Bull. Korean Chem. Soc 2007. 28, 477 .

4. Han. K. S.: Park. S. K.: Shim, S. Y.: Jahng. W. S.; Kim, N. J. Bull. Korean Chem. Soc. 1998. 19, 1165.

5. Cho. B. R.: Kim. Y. H.: Son1. K. W.: Khalil. C.: Kim. Y. H.: Teon1. S.-T. Bull. Korew Chent Soc. 2012. 23. 1253.

6. Moonl. J. R.: Kiml. B. S.: Kim. J-H. Bull. Korean Chent. Soe. 2006. 27,981

7. Kim. M. H.: Jin. J.-I: Lee, C. J.: Kim. N. J.: Park, C. H. Bull. Korean Chem. Soc. 2002. 23, 964 .

8. Lee. T.-H.: Lee. K.-S. Bull. Korean Chem. Soc. 2000. 21. 847.

9. Lee. S.-H.: Kim. Y.-K.: Won. Y.-H. Macronolecules 1999. 32. 342 .

10. Renate, D.: Walter. M. F. F.; Dietmar, K.: Hans, J. Dyes and Pignents 1997.34. 13.

11. Ambrosanio, P.: Centore. R:- Concilio, S.; Panunzi. B.: Sirigu. A.; Tirelli. N. Polvmer 1999. 40.4923.

12. Lee. T-Y.: Tung. W.-T.: Lee. W-T. Polnt Int 2006. 55.248.

13. Lee. J-Y.: Kim. T-H.: Jung. W.-T. Bull. Korem Chem. Soc. 2007. 28. 329 .

14. Cisneros, J. I. Appl. Opt. 1998. 37. 5262.

15. Herman. W. N.; Hayden, L. M. J. Opt. Soc. Am. B 1995. 12. 416. 